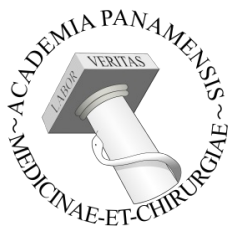

\title{
Caso de Interés Clínico
}

Microabscesos hepáticos en una paciente con síndrome antifosfolipidos. Reporte de caso. Liver microabscesses in a patient with antiphospholipid syndrome. Case report.

\author{
Araque Jonathan*, Umaña Kevin*, Padilla Erika**, Varela Lourdes*** \\ ${ }^{*}$ Facultad de postgrado médico-quirúrgica universidad libre sede Barranquilla. ${ }^{* *}$ Asesora científica. Facultad de postgrado mé- \\ dico-quirúrgica universidad libre sede Barranquilla. ${ }^{* * *}$ Asesora metodológica. Facultad de postgrado médico-quirúrgica universi- \\ dad libre sede Barranquilla
}

Palabras claves: microabscesos, síndrome antifosfolipidos, enzimas hepáticas, sepsis, vía biliar.

Key words: microabscesses, antiphospholipid syndrome, liver enzymes, sepsis, bile duct

\section{Correspondencia:}

Dr. Jonathan Araque L. Dr. Kevin Umaña C.

\section{Correo electrónico:} jona_arake@hotmail.com, kevingumana05@hotmail.com

Recibido: 20 de noviembre de 2019 Aceptado: 25 de febrero de 2020 Publicación: 27 de marzo de 2020

Conflicto de interés: Los autores declaran que no existe conflicto de interés alguno asociado en la publicación del presente trabajo.

\section{Resumen:}

Los abscesos hepáticos en la actualidad se siguen considerando un reto diagnóstico. Estos pueden dividirse en tres categorías principales según las condiciones subyacentes: infecciosas, malignas e iatrogénicas. Incluyen aquellos secundarios a la extensión directa de una infección local, bacteriemia sistémica e infecciones intraabdominales procedente de la porta, Sin embargo, a lo largo de los años, con los estudios diagnósticos la lista de factores de riesgo aumento, obligando a mas investigaciones para su entendimiento; logrando su pronto reconocimiento y tratamiento eficaz con el fin de obtener buenos resultados. Se presenta un caso de femenino con antecedentes de síndrome antifosfolípidos con dolor abdominal asociado a intolerancia a la vía oral. Imagenología abdominal muestra lesiones compatibles con microabscesos hepáticos siendo imposible la toma de muestra, requiriendo cubrimiento antibiótico de amplio espectro con resolución clínico radiológico completa. Tac de abdomen que muestra lesiones hepáticas múltiples con discreta colestasis intrahepática, lesiones compatibles con microabscesos múltiples.

\section{Abstract:}

Liver abscesses are currently still considered a diagnostic challenge. These can be divided into three main categories according to the underlying conditions: infectious, malignant and iatrogenic. They include those secondary to the direct extension of a local infection, systemic bacteraemia and intra-abdominal infections from the portal, however, over the years, with diagnostic studies the list of risk factors increased, forcing more research for its understanding; achieving its prompt recognition and effective treatment in order to obtain good results. A case of a female with a history of antiphospholipid syndrome with abdominal pain associated with oral intolerance is presented. Abdominal imaging shows lesions compatible with hepatic microabscesses, the sampling being impossible, requiring broad-spectrum antibiotic coverage with complete radiological clinical resolution. Abdominal tac showing multiple liver lesions with discrete intrahepatic cholestasis, lesions compatible with multiple microabscesses.

\section{INTRODUCCIÓN}

Los abscesos hepáticos se definen como colecciones focales, supurativas, únicas o múltiples, de tamaño variable, cuya mortalidad [11] es promedio es del 2-12 \% [1], según sea su presentación. Los agentes patógenos responsables de esta entidad pertenecen principalmente a dos grupos, bacterias y parásitos (Entamoeba histolytica) [2], y dan lugar a dos tipos de abscesos hepáticos: piógenos y amebianos con una incidencia va de 2,3 casos por 105 y 0,1 casos por 105 habitantes/año respectivamente [1].
No hay una característica clínica patognomónica en la identificación de la etiología de los abscesos hepáticos; sin embargo hay algunos hallazgos clínicos que pueden sugerir etiología de los mismos, por ejemplo la presencia de múltiples abscesos, de presentación en cualquier lóbulo hepático aumenta la probabilidad que sea piógeno[3], a diferencia de la existencia de lesiones únicas, de predominio en lóbulo hepático derecho, que es más característico de abscesos de origen amebiano[1][3], cabe resaltar que el punto de partida de este tipo de infección en el hígado puede provenir de áreas contiguas como la vía biliar en donde hay una alta predisposición en aque- 
llos pacientes con obstrucción de la misma o extensión directa de infecciones provenientes otra zona intraabdominal cercana o de la vía biliar per se; traumatismos, o secundarios a bacteriemia que por vía hematógena (cualquier bacteriemia sistémica, La pileflebitis [4], debida a diverticulitis [5], pancreatiti s[6], onfalitis [7], enfermedad inflamatoria intestinal[8] sea desde la arteria hepática o vena porta permiten el ingreso de gérmenes al parénquima hepático y en un gran número de casos ser criptogénico o con una etiología realmente establecida [3, 9,10,11].

Se ha documentado en el tiempo algunas predisposiciones como padecer de diabetes mellitus [11], manipulación quirúrgica del hígado o vía biliar, situaciones de inmunodepresión [12], ser portador de trasplante de hígado o la presencia de tumores malignos [9], en donde se evidencian frecuentemente la etiología piógena [3,9]; por otro lado, hay enfermedades en las que no se ha descrito hasta el momento asociación patogénica causal, como es el caso de las enfermedades autoinmunes, de estas enfermedades autoinmunes se cita en esta revisión el síndrome antifosfolipidos(SAF); el cual se caracteriza por la coexistencia de trombosis (venosa o arterial) o las pérdidas fetales recurrentes con la presencia de anticuerpos antifosfolipidos (anti B2 glicoproteina1, anticardiolipina, anticoagulante lupico). EI SAF puede ser un diagnostico aislado (SAF primario) o asociarse al lupus eritematoso sistémico (LES) u otra enfermedad reumática.

Dentro de los diagnósticos diferenciales [9], cabe citar al pseudotumor inflamatorio, hiperplasia nodular regenerativa las cuales tienen algo en común, que pueden caracterizarse por ser lesiones múltiples, en ocasiones difusas especialmente la hiperplasia nodular regenerativa y que se encuentran vinculadas con otros trastornos inflamatorios como enfermedades autoinmunes.

El tratamiento de los microabscesos hepáticos está basado en la terapia antibiótico [3] sí los abscesos son múltiples [12], solo hay que aspirar los de mayor tamaño, pero en caso que las lesiones sean pequeñas [10] $(<3 \mathrm{~cm})$ suelen curar con tratamiento antibiótico, [3] sin necesidad de drenaje y con relación a la presencia de síndrome antifosfolipidos, el tratamiento se basa en anticoagulación indefinida, partiendo de un antecedente previo de eventos trombótico arteriales o venosos.

El objetivo de este trabajo es describir la presentación, comportamiento y características clínicas, de una paciente con historia previa de síndrome antifosfolipidos primario que ingresa a la urgencia con dolor abdominal secundario a la presencia de microabscesos hepáticos, sin evidencia de una infección orgánica adyacente, o algún otro factor de riesgo de predisposición y de esta manera discutir la correlación causal entre ambas entidades teniendo en cuenta lo poco habitual del contexto de este cuadro.

\section{Presentación del Caso}

Se trata de paciente femenino de 31 años de edad con antecedentes síndrome antifosfolipidos primario diagnos- ticado en el año 2014 la cual debutó con evento trombótico por lo que recibe hasta la fecha rivaroxaban $20 \mathrm{mg}$ vía oral día. Paciente ingresa al servicio de urgencias por presentar cuadro clínico de 10 días de evolución consistente en dolor abdominal tipo urente irradiado región lumbar derecha y en forma de cinturón, intensidad 7/10 acompañado de náuseas y vómitos sin fiebre.

Al examen físico abdomen con dolor a la palpación en hipocondrio derecho y mesogastrio, sin signos de irritación peritoneal, ni masas perceptibles al examen físico, es valorada por el servicio de medicina interna descartando enfermedades autoinmunes asociadas a su enfermedad de base.

Paraclínicos reportan al momento del ingreso hemoglobina 10.4 (normal 12.6 - 16) normocítica normocrómica plaquetas $79.000 \mathrm{~mm} 3$ (normal 150.000 -450.000) bilirrubinas normales na 136 k 3,2 albumina 2.63 glucosa 125,6 C3 102mg/dl (valores normales 90-180) c4 13,2 (normal 10-40) creatinina 0,62 mg/dl (normal 0.67-1.17) got $279 \mathrm{mg} / \mathrm{dl}$ (normal 0-40) gpt $253 \mathrm{mg} / \mathrm{dl}$ (normal 0-41) ggt $301 \mathrm{mg} / \mathrm{dl}$ (normal 8-61 tp 11,9 inr 1,12 tpt 40 seg control 28,2 Hemocultivo x 2 negativo amonio $41 \mathrm{mg} / \mathrm{dl}$ (normal 16-50) proteínas totales $5,23 \mathrm{gr} / \mathrm{dl}$ (normal 6,48,3 ) albumina 2,36 gr/dl (normal 3,97-4,94) globulina 2,87 $\mathrm{gr} / \mathrm{dl}$ (1.5-3.0) amilasa $35 \mathrm{mg} / \mathrm{dl}$ (normal (13-60) lipasa $21,5 \mathrm{mg} / \mathrm{dl}$ (normal 28-100) alfafetoproteína 0,50 (normal 0 - $5 \mathrm{Ul} / \mathrm{ml}$ ) VIH 1 Y 2 acs negativo HVC-Ac negativo HBs-Ag negativo fosfatasa alcalina $193 \mathrm{mg} / \mathrm{dl}$ (normal 40120) GGT 288 (normal 8-61) anticoagulante lúpico IgG 58 positivo antifosfolipido IgM 6,44 negativo pruebas treponémicas no reactivo ASMA IgG 9,11 negativo (normal 0-20) AMA IgM 3,5 negativo (normal 0-20) antiro/ssa 2,28 (normal 0-20) antila/ssb 2,9 (normal 0-20) urea 16 (normal 16,6-48,5) creatinina 0.45 (normal 0,67-1.17) CA 19,9: 6,4 (normal 0,00-3,00) CA 15,3: 10.5 (normal 0.037,0) CA 125: 49,1 (normal 0,0-34,5) ACE 0,20 (normal fumador 0,00 - 5,00 no fumadores: $0,00-6,50$ )

Ecografía de abdomen total sin evidencia de sombra acústica compatible con litos en vesícula, engrosamiento de paredes u otras lesiones en cavidad abdominal, y doppler portal sin hipertensión, flujo hepatopetal.

14-12/19 Rx de tórax: Silueta cardiaca magnificada por posición en decúbito, no hay consolidaciones pulmonares, espacios pleurales libres, elementos de monitoreo externo.

16-12-18: Tac dinámico de hígado de 3 fases: conclusión: lesiones hepáticas múltiples con discreta colestasis intrahepática compatibles con microabscesos múltiples, pequeño derrame pleural bilateral.

20-12-18: doppler protal: no hay datos de hipertensión portal, ni degeneración cavernomatosa de la porta ni formación de colaterales portosistémicas.

21-12-18: colangioresonancia: pequeño derrame pleural derecho. resto normal

24-12-18: Angiotac de abdomen: Descarta presencia de trombos en circulación arterial y venosa del abdomen. 
Teniendo en cuenta la escasa mejoría, se decide ampliar estudios imagenológico con tomografía de abdomen y colangioresonancia compatibles con múltiples microabscesos hepáticos en ambos lóbulos, a su vez descartándose causas obstructivas, dado el tamaño y características de las lesiones con tendencia a confluir no era factible la realización de aspirado y/o lavado requiriendo por lo que se indicó manejo antibiótico inicialmente metronidazol y ceftriaxona, pero por escasa mejoría del cuadro clínico de la paciente teniendo en cuenta aparición respuesta inflamatoria sistémica, se considera infección complicada por esta razón se indica cubrimiento antibiótico de amplio espectro a meropenem con mejoría del cuadro clínico e imagenológico.

\section{DISCUSIÓN}

Los abscesos hepáticos pueden tener una presentación variable, según sea su etiología, y sus factores de riesgo asociados. Sin embargo, es curioso encontrar esta presentación inusual en forma de microabscesos, en el con- texto de un paciente cuyo único antecedente es el síndrome de anticuerpos antifosfolipidos.

Este reporte de caso podría corresponder al primer caso reportado en nuestra región de microabscesos hepáticos en paciente con síndrome antifosfolipidos, en quien, dada la presentación clínica por el deterioro agudo del estado general, evidencia de múltiples lesiones hepáticas por ultrasonografía con características imagenologicas típicas para microabscesos y mejoría tras instauración de terapia antibiótica hacen pensar en una etiología bacteriana de la misma.

Se desconoce si realmente el síndrome antifosfolipidos en ausencia de trombosis es una condición suficiente para ser considerada un factor de predisposición para el desarrollo de abscesos hepáticos. Se requerirán más estudios con el fin de determinar su asociación causal.

\section{CONCLUSIÓN}

Se presenta el caso de microabscesos hepático en paciente con síndrome antifosfolípidos, lo cual es muy inusual.

\section{REFERENCIAS}

[1] Torre A. Abscesos hepáticos. 2006;5:201-7.

[2] Leder AK, Weller PF. Entebeba histolytica extraintestinal amebiasis. 2019;1-18.

[3] Kim AY, Chung YRT, Capítulo ÍDEL, Hígado AL. Capítulo 84 - Infecciones bacterianas, parasitarias y fúngicas del hígado, incluidos los abscesos hepáticos [Internet]. Tenth Edit. Sleisenger y Fordtran. Enfermedades digestivas y hepáticas. Elsevier Espa8\#241;a, S.L.U.; 2018. 1374-1392 p. Available from: http://dx.doi.org/10.1016/B978-84-9113-2110/00084-X

[4] Correa S, Valiño J, Dufrechou C. Pileflebitis: Un desafío diagnóstico. Arch Med Interna. 2015;37(3):1446.

[5] Picón-Molina H, Pugliese A, Viscido G, Palencia R, Doniquian A. Diverticulitis de la flexura hepática del colon. Rev Chil cirugía. 2013;65(1):50-3.

[6] Quintas Lorenzo P, Cubiella Fernández J, Trillo Lista M, Fernández-Carrera Soler JM, Froján Parga P, Fernández Seara J. Absceso hepático y fístula biliar como complicaciones locales de una pancreatitis aguda: abordaje y tratamiento. Gastroenterol Hepatol. 2009;32(6):401-5.
[7] Baeza-Herrera C, López-Medina P, Nájera-Garduño HM, Martínez-Leo BA. Onfalopileflebitis y absceso hepático neonatal. Acta Pediátrica México. 2015;36(3):177.

[8] Galván A, Larrañaga E, Figueroa JM. Absceso hepático piogénico como complicación de la enfermedad de Crohn. 2000; 17:657-9.

[9] Mavilia MG, Molina M, Wu GY. The Evolving Nature of Hepatic Abscess: A Review. J Clin Transl Hepatol. 2016;4(2):158-68.

[10] Davis AJ, Mcdonald M. Absceso hepático piógeno. 2019;1-24.

[11] Chen SC, Tsai SJ, Lee YT, Yen CH, Huang CC, Lin $\mathrm{DB}$, et al. Predictors of mortality in patients with pyogenic liver abscess. Neth J Med. 2008;66(5):196203.

[12] Eroles Vega G, Mancebo Plaza AB, Fernández García C, Riva Jiménez I de la, Mecina Gutiérrez AB. Abscesos hepáticos: análisis retrospectivo de 68 casos. An Med Interna. 2010;25(7):335-41. 